\author{
Samuele Cortese \\ Eric Konofal \\ Bernardo Dalla Bernardina \\ Marie-Christine Mouren \\ Michel Lecendreux
}

\section{Sleep disturbances and serum ferritin levels in children with attention-deficit/ hyperactivity disorder}

Received: 16 March 2008

Accepted: 24 November 2008

Published online: 5 February 2009

S. Cortese, MD - E. Konofal, MD, PhD

M.-C. Mouren, MD · M. Lecendreux, MD

AP-HP, Child and Adolescent

Psychopathology Unit

Robert Debré Hospital

Paris VII University

Paris, France

S. Cortese, MD - B.D. Bernardina, MD

Department of Mother-Child and

Biology-Genetics

Child Neuropsychiatry Unit

G.B. Rossi Hospital

Verona University

Verona, Italy

E. Konofal, $\mathrm{MD}, \mathrm{PhD} \cdot \mathrm{M}$. Lecendreux, $\mathrm{MD}$ Pediatric Sleep Disorders Center

Robert Debré Hospital

Paris, France

E. Konofal, $\mathrm{MD}, \mathrm{PhD}$

Sleep Disorders Center

Pitié-Salpêtrière Hospital

Paris, France

S. Cortese, MD ( $\square)$

Service de Psychopathologie

de l'Enfant et de l'Adolescent

Hôpital Robert Debré

48 Boulevard Sérurier

75019 Paris, France

Tel.: +33-1/40032263

Fax: +33-1/40032297

E-Mail: samuele.cortese@gmail.com
Abstract Background A subset of children with attention-deficit/ hyperactivity disorder (ADHD) may present with impairing sleep disturbances. While preliminary evidence suggests that iron deficiency might be involved into the pathophysiology of daytime ADHD symptoms, no research has been conducted to explore the relationship between iron deficiency and sleep disturbances in patients with ADHD. The aim of this study was to assess the association between serum ferritin levels and parent reports of sleep disturbances in a sample of children with ADHD. Methods Subjects: Sixty-eight consecutively referred children (6-14 years) with ADHD diagnosed according to DSM-IV criteria using the semistructured interview Kiddie-

SADS-PL. Measures: parents filled out the Sleep Disturbance Scale for Children (SDSC) and the Conners Parent Rating Scale (CPRS). Serum ferritin levels were determined using the Tinaquant method. Results Compared to children with serum ferritin levels $\geq 45 \mu \mathrm{g} / \mathrm{l}$, those with serum ferritin levels $<45 \mu \mathrm{g} / \mathrm{l}$ had significantly higher scores on the SDSC subscale "Sleep wake transition disorders" (SWTD) $(P=0.042)$, which includes items on abnormal movements in sleep, as well as significantly higher scores on the CPRS-ADHD index $(P=0.034)$. The mean scores on the other SDSC subscales did not significantly differ between children with serum ferritin $\geq 45$ and $<45 \mu \mathrm{g} / \mathrm{l}$. Serum ferritin levels were inversely correlated to SWTD scores $(P=0.043)$. Conclusion Serum ferritin levels $<45 \mu \mathrm{g} / \mathrm{l}$ might indicate a risk for sleep wake transition disorders, including abnormal sleep movements, in children with ADHD. Our results based on questionnaires set the basis for further actigraphic and polysomnographic studies on nighttime activity and iron deficiency in ADHD. Research in this field may suggest future trials of iron supplementation (possibly in association with ADHD medications) for abnormal sleep motor activity in children with ADHD.

Key words ADHD sleep - iron deficiency 


\section{Introduction}

Attention-deficit/hyperactivity disorder (ADHD) is one of the most common childhood neurobehavioral disorders, estimated to affect $5-8 \%$ of school-aged children worldwide [4]. According to the Diagnostic and Statistical Manual of Mental Disorders, 4 th edition, Text Revision (DSM-IV-TR) [3], ADHD is defined by a persistent, pervasive, and age-inappropriate pattern of inattention, hyperactivity-impulsivity or both.

Although quite overlooked by child psychiatrists and pediatricians, in clinical practice sleep disturbances are frequently reported in children referred for ADHD symptoms $[7,25]$. According to a meta-analysis by Cortese et al. [8], children with ADHD have significantly higher daytime sleepiness, apnea-hypopnea indexes, and more movements in sleep compared with controls. This suggests that ADHD may not be limited to daytime, but it is likely to be associated with both daytime and nighttime impairment, at least in a subset of patients.

In a case-control study, Konofal et al. [18] found significantly lower serum ferritin levels in children with ADHD compared to controls, suggesting that iron deficiency might be involved into the pathophysiology of ADHD. Konofal et al. [18] also found a significant inverse correlation between ADHD severity and serum ferritin levels, pointing to an association between daytime ADHD symptoms and iron deficiency. A significant inverse correlation between ADHD symptoms and serum ferritin levels was replicated in the study by Oner and Oner [23].

While we begin to gain insight into the role of iron deficiency in daytime ADHD symptoms, limited research has been conducted to explore the relationship between iron deficiency and sleep disturbances in patients with ADHD.

Evidence from magnetic resonance imaging [15] and autopsy data [1], as well as from intravenous iron trials $[13,14,16]$, supports the role of iron deficiency in the pathophysiology of two motor disorders which may affect sleep initiation and/or continuity in adults: restless legs syndrome (RLS) and periodic limb movement disorder in sleep (PLMD). (PLMD is diagnosed when the following conditions are present: (1) periodic limb movements in sleep exceeding norms for age, (2) clinical sleep disturbance, and (3) the absence of another primary sleep disorder or reason for the periodic limb movements in sleep, including RLS [2]).

Available studies in children with ADHD have confirmed a significant association between iron deficiency and RLS [19, 24] as well as PLMD [28].

However, no study has assessed the relationship between iron status and more global motor activity in sleep or other sleep alterations in children with ADHD.
Interestingly, a recent study by Dosman et al. [11] suggested a relationship between restless sleep and iron deficiency in children with autism spectrum disorder. Another recent study by Peirano et al. [26] also showed altered sleep spindle patterns in 6month-old infants with iron-deficiency anemia.

On the basis of these recent findings, we wondered whether iron deficiency in patients with ADHD may be associated not only with daytime symptoms, but also with abnormal motor activity in sleep and, possibly, with other sleep alterations.

In the present study we felt it interesting to focus on the parental perception of sleep problems. Given the role of the parents as informants during the clinical consultation, we believe that this aspect may be of interest in the clinical practice.

Therefore, the aim of this study was to assess the relationship between iron deficiency (estimated by serum ferritin levels) and parent reports of sleep disturbances (including abnormal motor activity in sleep) in a sample of children with ADHD.

After an accurate revision of the literature on the topic, we decided to use a serum ferritin cut-off $=$ $45 \mu \mathrm{g} / \mathrm{l}$ to estimate iron deficiency. As correctly pointed out by Dosman et al. [10], the cut-off value used for low ferritin differs across centers, limiting its accuracy for detecting the presence of iron deficiency. The World Health Organization reports a mean of normal ferritin levels of $34 \mu \mathrm{g} / \mathrm{l}$ [9]. Milman et al. [22] reported a mean of $38.5 \mu \mathrm{g} / \mathrm{l}$. The American Association for Clinical Chemistry uses a range of $6-24 \mu \mathrm{g} / \mathrm{l}$ (1-5 years) and 10-55 $\mu \mathrm{g} / \mathrm{l}$ (6-9 years) [29]. Picchietti [27] has correctly suggested that the issue is what level of iron deficiency might have physiological significance. When iron stores are depleted, typically below the widely accepted cut-off for serum ferritin of $12 \mu \mathrm{g} / \mathrm{l}$, there is decreased hemoglobin synthesis [27]. However, peripheral iron stores are not fully replenished until a much higher serum ferritin level is present [27]. The movement of iron from the peripheral stores into the central nervous system (CNS) may be influenced by the degree of peripheral iron store saturation. Thus, a "normal" ferritin level for hemoglobin and myoglobin synthesis could be insufficient for neurotransmitter function in the brain. In line with these observations, in a review of the literature on the topic, Allen and Earley [1] pointed out that the usually published normal values for ferritin tend to confuse iron status evaluations since they represent population samples, not biological, norms. These authors suggest a cut-off of about $45 \mu \mathrm{g} / \mathrm{l}$ to indicate low peripheral iron stores. Therefore, in the present study we considered that a serum ferritin cut-off $=45 \mu \mathrm{g} / \mathrm{l}$ was more appropriate than lower cut-off values used in other previous studies. 


\section{Methods}

\section{Subjects}

We performed a retrospective chart review of patients consecutively referred to the ADHD outpatient clinic of the Child and Adolescent Psychopathology Service at the University Hospital Robert Debré in Paris, France, from November 2006 to November 2007. In the present study, we examined data relative to patients aged 6-14 years and diagnosed with ADHD according to DSM-IV-TR criteria. Data from patients referred for symptoms of inattention, impulsivity, and/or hyperactivity not meeting full DSM criteria were not analyzed.

\section{Diagnosis and assessment of ADHD}

Diagnosis of ADHD, as well as of comorbid psychiatric disorders, was based on separate clinical interviews with the child and his parents. Information from teachers and other relevant sources was also obtained. The diagnosis of ADHD was confirmed using the Kiddie-SADS-PL [17], which is a semistructured diagnostic interview designed to assess current and past episodes of psychopathology in children and adolescents according to DSM-III-R and DSM-IV criteria. It is administered by interviewing the parent(s), the child, and finally achieving summary ratings which include all sources of information (parent(s), child, school charts, and other).

The severity of ADHD symptoms was assessed using the Conners Parent Rating Scale (CPRS) [6]. It is a widely used questionnaire which assesses ADHD symptoms. We used the CPRS-48 items. It provides an ADHD-index score which covers symptoms of hyperactivity, impulsivity and inattention, as well as the following subscales: conduct problems, learning problems, psychosomatic problems, impulsivehyperactive behaviors, and anxiety. For the purposes of this study, we analyzed the scores on the ADHDindex (CPRS-ADHD index). The CPRS is the only ADHD rating scale translated into French and validated on a French childhood population [12].

\section{Assessment of sleep disturbances}

During the first visit with patients referred for a potential diagnosis of ADHD, we systematically ask the parents to fill out the Sleep Disturbance Scale for Children (SDSC) by Bruni et al. [5]. The SDSC assesses sleep behaviors and disturbances during the previous six months. It includes 26 items. The SDSC consists of six factors which represent the most common areas of sleep disorders in childhood and adolescence: difficulty in initiating and maintaining sleep (DIMS), sleep breathing disorders (SBD), arousal disorders (DA), sleep-wake transition disorders (SWTD), disorders of excessive somnolence (DOES), and sleep hyperhydrosis (SHY). The total score (TOT) is the sum of the 26 items. In particular, the SWTD subscale includes the following items: "the child startles or jerks parts of the body while falling asleep"; "the child shows repetitive actions such as rocking or head banging while falling asleep"; "the child experiences vivid dream-like scenes while falling asleep"; "the child has frequent twitching or jerking of legs while asleep or often changes position during the night or kicks the covers off the bed"; "you have observed the child talking in his/her sleep"; "the child grinds teeth during sleep". The DA subscale includes the following items: "you have observed the child sleepwalking"; "the child wakes from sleep screaming or confused so that you cannot seem to get through to him/her, but has no memory of these events the next morning"; "the child has nightmares which he/she does not remember the next day". The SDSC has shown high internal consistency in controls (0.79) and sleep disorder subjects (0.71), as well as adequate test/ retest reliability for the total $(r=0.71)$ and single item scores.

SDSC scores were available for all the patients included in the study.

\section{Assessment of iron deficiency}

Since we are involved in research on iron metabolism in children with ADHD, we systematically propose a measure of hemoglobin, serum iron and ferritin levels to patients referred for a potential diagnosis of ADHD. All the children included into this study had a complete blood count and measurement of serum ferritin levels, as well as of serum iron and hemoglobin. Serum ferritin and iron levels were determined with commercial kits: ferritin levels by the Tinaquant method and iron levels by the ferrozine method (Roche, Basel, Switzerland). The blood samples were collected in the morning for all the patients.

Our research on iron and ADHD has been approved by the local ethics review board (Pitié-Salpêtrière Hospital, Paris).

\section{Statistical analysis}

Demographic and clinical data were shown as means and SD or percentages. Given that data were not normally distributed, the non parametric test MannWhitney $U$ was used to compare the mean scores on each subscales of the SDSC, as well as on the CPRS- 
Table 1 Demographic and clinical characteristics of the subjects. Mean and SD (or percentages)

\begin{tabular}{llll}
\hline & Males & Females & All \\
\hline$N$ & 56 & 12 & 68 \\
Age (years) & $8.5(2.3)$ & $10.1(2.7)$ & $9.1(2.4)$ \\
Race (\%) & & & \\
Caucasian & $46(82.1)$ & $10(83.3)$ & $56(82.3)$ \\
African & $8(14.1)$ & $2(16.6)$ & $10(17.8)$ \\
Asian & $2(3.5)$ & $0(0)$ & $2(3.5)$ \\
ADHD-I & $13(23.2)$ & $7(58.3)$ & $20(29.4)$ \\
ADHD-C & $36(64.2)$ & $4(33.3)$ & $40(51.4)$ \\
ADHD-HI & $7(12.5)$ & $2(16.6)$ & $9(13.2)$ \\
Comorbid psychiatric disorders (\%) & & \\
ODD & $32(57.1)$ & $1(8.3)$ & $33(48.5)$ \\
CD & $2(3.5)$ & $0(0)$ & $2(2.9)$ \\
SAD & $2(3.5)$ & $1(8.3)$ & $3(4.4)$ \\
GAD & $3(5.3)$ & $5(41.6)$ & $8(11.76)$ \\
P & $5(8.9)$ & $1(8.3)$ & $6(8.8)$ \\
OCD & $2(3.5)$ & $1(8.3)$ & $3(4.41)$ \\
MDD & $1(1.7)$ & $1(8.3)$ & $2(2.9)$ \\
DYS & $4(7.1)$ & $2(16.6)$ & $6(8.8)$ \\
TS & $3(5.3)$ & $0(0)$ & $3(4.4)$ \\
TIC & $2(3.5)$ & $0(0)$ & $2(2.9)$ \\
Serum ferritin $(\mu g / l)$ & $38.9(26.7)$ & $24.2(13.0)$ & $38.5(26.7)$ \\
Serum iron $(\mu \mathrm{g} / \mathrm{l})$ & $97.4(13.9)$ & $87.2(25.5)$ & $95.2(16.9)$ \\
Hemoglobin $(g / \mathrm{dl})$ & $12.7(3.6)$ & $12.1(4.3)$ & $12.5(4.8)$ \\
\hline
\end{tabular}

ADHD-I ADHD predominantly inattentive type, ADHD-C ADHD combined type, $A D H D-H I A D H D$ predominantly hyperactive-impulsive type, $O D D$ oppositional defiant disorder, $C D$ conduct disorder, $S A D$ separation anxiety disorder, $G A D$ generalized anxiety disorder, $P$ phobia, $O C D$ obsessive compulsive disorder, MDD major depressive disorder, DYS dysthymic disorder, TS Tourette's syndrome, TIC tic disorder

ADHD index, between patients with serum ferritin levels $\geq 45$ and $<45 \mu \mathrm{g} / \mathrm{l}$. Spearman correlations and multiple linear regression were used to assess the association between serum ferritin levels and scores on the SDSC as well as on the CPRS-ADHD index. The scores on the SDSC and the serum ferritin levels in three ADHD types (inattentive, hyperactiveimpulsive, and combined) were compared using the Kruskal-Wallis test. A probability level of $P<0.05$ was used to indicate statistical significance. All statistical analyses were performed using the SPSS v. 13.0 software for Windows package for personal computers (SPSS, Inc., Chicago, IL, USA).

\section{Results}

Sixty-eight patients were included into the study. The power analysis indicated that such a number of patients allowed us to detect a difference of about ten points in the SDSC T scores (beta: 0.05).

Table 1 shows the demographic and clinical characteristics of the subjects, including the mean serum iron and ferritin values, as well as hemoglobin levels. No patient presented with significant nutritional
Table 2 Mean values (SD) on the SDSC (Sleep Disturbance Scale for Children) and CPRS-index scores

\begin{tabular}{llll}
\hline SDSC & Males & Females & All \\
\hline DIMS & $63.1(17.5)$ & $75.5(21.3)$ & $65.6(16.6)$ \\
SBD & $52.2(13.8)$ & $67.1(13.4)$ & $55.5(12.3)$ \\
DA & $58.5(12.6)$ & $54.6(17.3)$ & $56.2(12.2)$ \\
SWTD & $62.1(15.3)$ & $65.3(17.0)$ & $63.7(17.6)$ \\
DOES & $56.6(16.4)$ & $55.3(15.9)$ & $56.9(12.7)$ \\
SHY & $56.6(13.2)$ & $52.2(15.2)$ & $55.1(11.1)$ \\
TOT & $65.2(10.7)$ & $69.6(13.5)$ & $66.9(11.0)$ \\
CPRS-ADHD index & $76.0(11.3)$ & $76.2(8.5)$ & $76.3(11.3)$
\end{tabular}

DIMS difficulty in initiating and maintaining sleep, $S B D$ sleep breathing disorders, DA arousal disorders, SWTD sleep-wake transition disorders, DOES disorders of excessive somnolence, SHY sleep hyperhydrosis. Values on the SDSC and CPRS-ADHD index are expressed as $T$ scores

Table 3 Multiple regression analysis: final model

\begin{tabular}{|c|c|c|c|c|}
\hline & $R^{2}$ & Beta coefficient & SE & $P$ \\
\hline $\begin{array}{l}\text { Dependent variable: serum } \\
\text { ferritin }\end{array}$ & 0.138 & & & \\
\hline \multicolumn{5}{|l|}{ Independent variables } \\
\hline SWTD & & -0.238 & 0.154 & 0.043 \\
\hline CPRS-ADHD index & & -0.245 & 0.305 & 0.037 \\
\hline
\end{tabular}

problems as reported by parents. Accurate examination of patients' clinical records confirmed this finding. No subject presented with anemia, infection, other inflammatory condition or any other significant medical conditions. Of note, all patients were treatment naive when the assessment was carried out. The examination of clinical records allowed us also to assess the sleeping conditions of the subjects. Only two children presented with co-sleeping and three with bedroom sharing.

Table 2 reports the mean $T$ scores on the SDSC and on the CPRS-ADHD index.

Forty-one patients $(60 \%)$ presented with serum ferritin levels $<45 \mu \mathrm{g} / \mathrm{l}$.

Mann-Whitney $U$ test showed that patients with serum ferritin levels $<45 \mu \mathrm{g} / \mathrm{l}$ had significantly higher scores on the subscale "SWTD" of the SDSC $(P=0.042)$, as well as on the CPRS-ADHD index $(P=0.034)$, than those with serum ferritin levels $\geq 45 \mu \mathrm{g} / \mathrm{l}$. The mean scores on the other subscales of the SDSC did not significantly differ between the two groups $(P>0.05)$. Serum ferritin levels were inversely correlated to the scores SWTD of the SDSC $(P=0.032)$, as well as to the CPRS-ADHD index $(P=0.023)$. The multiple linear regression using the serum ferritin levels as dependent variable and the SWTD and the CPRS-ADHD index as independent variables, showed that both SWTD and CPRS-ADHD index were independently associated with serum ferritin levels (Table 3 ). 
The scores on the SDSC and the serum ferritin levels were not significantly different in three ADHD types $(P>0.05)$.

\section{Discussion}

To our knowledge, this is the first study that assessed the relationship between serum ferritin levels and sleep disturbances in children with ADHD.

The major finding of the study was that children with serum ferritin $<45 \mu \mathrm{g} / \mathrm{l}$, compared to children with serum ferritin $\geq 45 \mu \mathrm{g} / \mathrm{l}$, had significantly higher scores not only on the CPRS-ADHD index, but also on the subscale "sleep wake transition disorders" of the SDSC. Moreover, there was a significant inverse correlation between the serum ferritin levels and the SWTD scores of the SDSC. SWTD scores were significantly associated with the serum ferritin levels after controlling for the CPRS-ADHD index in the regression model.

Interestingly, most of the items of the SWTD are related to abnormal movements. Therefore, we hypothesize that serum ferritin levels $<45 \mu \mathrm{g} / \mathrm{l}$ indicate a risk for abnormal motor activity in sleep in children with ADHD. Clearly, the cross-sectional nature of this study can only suggest a significant relation but cannot prove causality between low serum ferritin levels and abnormal movements in sleep.

As previously stated, the choice of the cut-off used to indicate iron deficiency (serum ferritin levels $<45 \mu \mathrm{g} / \mathrm{l}$ ) was made according to Picchietti [27] as well as Allen and Earley's [1] suggestions in order to consider a cut-off with a potential physiological relevance. Indeed, the results of the present study did confirm that a ferritin cut-off $=45 \mu \mathrm{g} / \mathrm{l}$ is appropriate to assess the impact of iron deficiency on abnormal motor activity in sleep (as reported by parents).

In our sample, $60 \%$ of patients presented with serum ferritin levels $<45 \mu \mathrm{g} / \mathrm{l}$. Interestingly, Millichap et al. [21] found that $74 \%$ of their patients with ADHD had serum ferritin levels below $50 \mathrm{ng} / \mathrm{mL}$, which is quite similar to our findings.

The mechanisms by which iron deficiency may impact on global motor activity in sleep are still not clear. Since it has been demonstrated that iron deficiency alters dopaminergic functions and given that the dopaminergic system is involved in motor control [1], we speculate that a potential dysfunction of dopaminergic pathways may play a significant role, similarly to what has been hypothesized for RLS. Another interesting explication has been proposed by Peirano et al. [26], who suggested that the reduction in sleep spindles due to iron deficiency may lead to altered motor activity in sleep since sleep spindles have been associated with suppression in muscle tone.
The scores on the other subscales of the SDSC did not significantly differed between patients with serum ferritin levels $<45$ and $\geq 45 \mu \mathrm{g} / \mathrm{l}$. This may indicate that sleep disturbances (assessed by parents) other than altered motor activity in sleep may not be significantly influenced by iron deficiency or that the cut-off that we used was not appropriate to detect a significant difference in these subscales.

We found no significant differences in the SDSC scores among the three ADHD types, as well as no significant serum ferritin levels. However, it is possible that, given the limited number of subjects in the hyperactive-impulsive group $(N=9)$, our study was underpowered to detect significant differences.

Finally, the significant inverse correlation between the serum ferritin levels and the CPRS-ADHD index confirms the previous findings by Konofal et al. [18] obtained in a different sample of children with ADHD.

We think that our study has several strengths.

First, the sample was not exposed to significant selection bias, since we examined data from patients consecutively referred to a general ADHD consultation, without a selection for patients with comorbid sleep problems. Therefore, we are confident that our sample is representative of a typical clinical ADHD population addressed to a general child psychiatric or pediatric outpatient clinic. Of note, we included all consecutively referred children diagnosed with ADHD in the study period.

Second, all the patients of the study were rigorously diagnosed with ADHD according to state of the art assessment of this disorder (i.e. DSM criteria confirmed by semi- structured interviews taking into account several sources of information).

Third, we used a well validated questionnaire (SDSC) to asses sleep disturbances in children.

Fourth, no subjects presented with infection or other inflammatory condition. Since ferritin is a marker of inflammation, this is of particular relevance to our results.

However, some limitations should also be reported.

First, the sample size was relatively small.

Second, and most importantly, in this study we were interested in parental perception of sleep and we did not assess sleep alterations using objective measures (actigraphy, polysomnography, video analysis).

Third, the lack of a control group is a limitation of this study.

Therefore, we recognise that the results of our study should be considered with caution. However, we think that our results may be of clinical relevance, indicating that children with serum ferritin $<45 \mu \mathrm{g} / \mathrm{l}$ may represent a subgroup at risk for parentally reported abnormal motor activity in sleep. We also 
believe that our study sets the basis for further research exploring the potential association between serum ferritin levels and abnormal motor activity using objective measures of sleep (i.e. actigraphy and polysomnography).

Gaining insight into the role of iron deficiency in sleep disturbances may allow us to better understand the pathophysiology of sleep disturbances in these patients, suggesting potential effective treatment strategies. Simakajornboon et al. [28] reported a decrease in periodic limb movements in sleep in children treated with iron sulphate. However, no study has assessed the effect of iron supplementation on general motor activity during sleep in children with
ADHD. Since it has been reported a decrease of nighttime activity level in ADHD patients treated with stimulants (acting on the dopaminergic system) [20], and given that iron deficiency may impact on dopaminergic functions [30], it would be of interest to consider trials with ADHD medications plus iron supplementation for excessive nighttime activity levels in patients with ADHD.

Since sleep disturbances represent a source of distress for the child and his family and they may aggravate daytime ADHD symptoms [25], research on iron deficiency and sleep in ADHD is of clinical relevance and should be encouraged.

\section{References}

1. Allen RP, Earley CJ (2007) The role of iron in restless legs syndrome. Mov Disord 22:S440-S448

2. Allen RP, Picchietti D, Hening WA, Trenkwalder C, Walters AS, Montplaisir J (2003) Restless legs syndrome: diagnostic criteria, special considerations, and epidemiology. A report from the restless legs syndrome diagnosis and epidemiology workshop at the National Institutes of Health. Sleep Med 4:101-119

3. American Psychiatric Association (2000) Diagnostic and statistical manual of mental disorders, 4 th ed. Text revision

4. Biederman J (2005) Attention-deficit/ hyperactivity disorder: a selective overview. Biol Psychiatry 57:1215-1220

5. Bruni O, Ottaviano S, Guidetti V, Romoli M, Innocenzi M, Cortesi F, Giannotti F, The Sleep Disturbance Scale for Children (SDSC) (1996) Construction and validation of an instrument to evaluate sleep disturbances in childhood and adolescence. J Sleep Res 5:251-261

6. Conners CK, Barkley RA (1985) Rating scales and checklists for child psychopharmacology. Psychopharmacol Bull 21:809-843

7. Corkum P, Tannock R, Moldofsky H (1998) Sleep disturbances in children with attention-deficit/hyperactivity disorder. J Am Acad Child Adolesc Psychiatry 37:637-646

8. Cortese S, Konofal E, Yateman N, Mouren MC, Lecendreux M (2006) Sleep and alertness in children with attention-deficit-hyperactivity disorder: a systematic review of the literature. Sleep 29:504-511
9. DeMaeyer EM (1989) Preventing and controlling iron deficiency anaemia through primary health care-a guide for health administrators and programme managers. WHO, Geneva

10. Dosman CF, Drmic IE, Brian JA, Senthilselvan A, Harford M, Smith R, Roberts SW (2006) Ferritin as an indicator of suspected iron deficiency in children with autism spectrum disorder: prevalence of low serum ferritin concentration. Dev Med Child Neurol 48:1008-1009

11. Dosman CF, Brian JA, Drmic IE, Senthilselvan A, Harford MM, Smith RW, Sharieff W, Zlotkin SH, Moldofsky H, Roberts SW (2007) Children with autism: effect of iron supplementation on sleep and ferritin. Pediatr Neurol $36: 152-158$

12. Dugas M (1987) L' hyperactivitè chez l' enfant. PUF, Paris

13. Earley CJ, Heckler D, Allen RP (2004) The treatment of restless legs syndrome with intravenous iron dextran. Sleep Med 5:231-235

14. Earley CJ, Heckler D, Allen RP (2005) Repeated IV doses of iron provides effective supplemental treatment of restless legs syndrome. Sleep Med 6:301-305

15. Earley CJ, Barker B, Horska A, Allen RP (2006) MRI-determined regional brain iron concentrations in early- and lateonset restless legs syndrome. Sleep Med 7:458-461

16. Earley CJ, Horska A, Mohamed MA, Barker PB, Beard JL, Allen RP (2008) A randomized, double-blind, placebocontrolled trial of intravenous iron sucrose in restless legs syndrome. Sleep Med (in press)
17. Kaufman J, Birmaher B, Brent D, Rao U, Flynn C, Moreci P, Williamson D, Ryan N (1997) Schedule for affective disorders and schizophrenia for school-age children-present and lifetime version (K-SADS-PL): initial reliability and validity data. J Am Acad Child Adolesc Psychiatry 36:980-988

18. Konofal E, Lecendreux M, Arnulf I, Mouren MC (2004) Iron deficiency in children with attention-deficit/hyperactivity disorder. Arch Pediatr Adolesc Med 158:1113-1115

19. Konofal E, Cortese S, Marchand M, Mouren MC, Arnulf I, Lecendreux M (2007) Impact of restless legs syndrome and iron deficiency on attention-deficit/hyperactivity disorder in children. Sleep Med 8:711-715

20. Kooij JJ, Middelkoop HA, van GK, Buitelaar JK (2001) The effect of stimulants on nocturnal motor activity and sleep quality in adults with ADHD: an open-label case-control study. J Clin Psychiatry 62:952-956

21. Millichap JG, Yee MM, Davidson SI (2006) Serum ferritin in children with attention-deficit hyperactivity disorder. Pediatr Neurol 34:200-203

22. Milman N, Backer V, Laursen EM, Graudal N, Ibsen KK, Jordal R (1994) Serum ferritin in children, adolescents. Results from population surveys in 1979 and 1986 comprising 1312 individuals. Eur J Haematol 53:16-20

23. Oner P, Oner O (2008) Relationship of ferritin to symptom ratings children with attention deficit hyperactivity disorder: effect of comorbidity. Child Psychiatry Hum Dev 39:323-330

24. Oner P, Dirik EB, Taner Y, Caykoylu A, Anlar O (2007) Association between low serum ferritin and restless legs syndrome in patients with attention deficit hyperactivity disorder. Tohoku J Exp Med 213:269-276 
25. Owens J (2005) The ADHD and sleep conundrum: a review. J Dev Behav Pediatr 26:312-322

26. Peirano P, Algarin C, Garrido M, Algarin D, Lozoff B (2007) Iron-deficiency anemia is associated with altered characteristics of sleep spindles in NREM sleep in infancy. Neurochem Res 32:1665-1672
27. Picchietti D (2007) Is iron deficiency an underlying cause of pediatric restless legs syndrome and of attention-deficit/ hyperactivity disorder? Sleep Med 8:693-694

28. Simakajornboon N, Gozal D, Vlasic V, Mack C, Sharon D, McGinley BM (2003) Periodic limb movements in sleep and iron status in children. Sleep 26:735-738
29. Soldin SJ, Brugnara C, Hicks JM (1999) Pediatric reference ranges. American Association for Clinical Chemistry, Washington DC

30. Youdim MB, Ben-Shachar D, Ashkenazi R, Yehuda S (1983) Brain iron and dopamine receptor function. Adv Biochem Psychopharmacol 37:309-321 